\title{
Affleck-Dine baryogenesis in large extra dimensions
}

\author{
Anupam Mazumdar \\ The Abdus Salam International Centre for Theoretical Physics, I-34100, Trieste, Italy \\ A. Pérez-Lorenzana \\ The Abdus Salam International Centre for Theoretical Physics, I-34100, Trieste, Italy \\ and Departamento de Física, Centro de Investigación y de Estudios Avanzados del I.P.N., Apartado Postal 14-740, \\ 07000, México, Distrito Federal, México
}

(Received 23 March 2001; revised manuscript received 23 April 2001; published 24 April 2002)

\begin{abstract}
Baryogenesis in models in which the fundamental scale is as low as $1 \mathrm{TeV}$ in the context of large extra dimensions is a challenging problem. The requirement for the departure from thermal equilibrium necessarily ties any low-scale baryogenesis with that of a successful inflationary model, which automatically provides the out-of-equilibrium condition after the end of inflation. However, it is also noticeable that in these models the reheat temperature of the Universe is strongly constrained from the overproduction of Kaluza-Klein modes, which enforces a very low reheat temperature. In this paper, we describe a possible scenario for baryogenesis which has similar characteristics to an Affleck-Dine field. We notice that in order to have an adequate baryon to entropy ratio, one must to promote this Affleck-Dine field to reside in the bulk.
\end{abstract}

DOI: 10.1103/PhysRevD.65.107301

PACS number(s): $98.80 . \mathrm{Cq}, 11.10 . \mathrm{Kk}, 11.30 . \mathrm{Fs}$

\section{INTRODUCTION}

Baryogenesis is an interesting offshoot of cosmology and particle physics, which tries to explain why the ratio of baryon density and photon density is given by one part in $10^{10}$ during the nucleosynthesis era [1]. The synthesis of light elements depends crucially on this ratio which tells us that, in the absence of any observed antimatter regions, the baryon density should be equal to the cosmological baryon asymmetry. There are many proposals which can satisfy the three conditions: namely, $C$ and $C P$ violation, $B$ or $L$ violation, and out-of-equilibrium decay, which are the essential ingredients for baryogenesis [2]. Of the three mentioned conditions, the last one has to come purely from the cosmological evolution of the Universe. It is quite probable that the early Universe might have had a strong departure from thermal equilibrium due to a large expansion rate of the Universe and the presence of heavy decaying particles; however, this possibility gradually becomes difficult to acquire at scales which are comparable to the electroweak scale. As a second alternative, one might expect to attain the departure from thermal equilibrium via some phase transitions which would break global or gauge symmetry; a perfect example is an electroweak phase transition where there is an anomalous $B+L$ violation, for a review, see Ref. [3]. In the former situation, the departure from thermal equilibrium is usually connected with inflation. Inflation is an attractive paradigm which solves a range of troublesome problems of the big bang cosmology in addition to acting as the best candidate for producing almost scale invariant density perturbations. After a period of inflation, the Universe undergoes an era of reheating, and this is precisely where one might expect to produce massive bosons and their out-of-equilibrium decay, which might lead to the desired baryon to entropy ratio.

On the other hand, recent trends in solving the hierarchy problem, in the context of theories with extra dimension, suggest that the strength of the fundamental scale might be much lower than the four-dimensional Planck scale. If that scale is the electroweak scale, then the hierarchy between the Planck scale and the electroweak scale can be inverted by assuming that there exist large extra dimensions, which can be as large as $\mathrm{mm}$ [4]. It is also assumed that the standard model (SM) particles are trapped in a four-dimensional hypersurface (a 3-brane), thus they are not allowed to propagate in the whole higher-dimensional space (bulk). However, it is generically assumed that besides gravity, SM singlets may propagate in the bulk. Among them, the inflaton can be a candidate, which is less favored to be a brane field (see, for instance, Refs. [5,6]). However, in these models the Universe during the radiation-dominated epoch reaches its maximum temperature very close to $\mathrm{MeV}$, which we shall discuss in details in the coming sections. For such a low reheat temperature, baryogenesis is a challenging task for two reasons: (i) the late decay of particles including the inflaton, which is responsible for reheating the Universe, and (ii) the operators which might lead to baryon number violation must be suppressed due to stringent constraints on proton lifetime. This restricts us to a few choices of baryogenesis models which may work well in the presence of a small fundamental scale, such as $\sim O \quad(\mathrm{TeV})[7]$.

Another possibility may appear from the fact that reheat temperature is not the maximum temperature in the Universe after the end of inflation. Usually, reheating takes a while and it is possible to reach a temperature during the process of reheating which can be quite high, however this rise in temperature depends crucially on the scale when inflation comes to an end [8]. If this is the case, then it is quite possible that the rate of sphaleron transitions is active, even though the reheat temperature is much lower than $100 \mathrm{GeV}$ [9]. In this paper, we describe a completely different possibility. This mechanism does not depend on the predictability of a high rise in temperature during the reheating era. Our scheme is analogous to the Affleck-Dine (AD) mechanism of baryogenesis. We begin our paper with a brief discussion on the reheat 
temperature and its bounds. Then we describe the possibility of leptogenesis, which can be reprocessed into baryon number $B$ by anomalous $(B+L)$-violating sphaleron interactions, which otherwise preserve $B-L$. However, we also point out that there are many obstacles with this mechanism. Finally, we discuss baryogenesis by assuming a singlet carrying a global charge and decaying mainly into SM quarks and leptons to provide an adequate baryon to entropy ratio just at the end of reheating. Finally, we conclude our paper by summarizing the facts.

\section{REHEAT TEMPERATURE OF THE UNIVERSE}

In models with large extra dimensions, the reheat temperature is constrained from the possible thermal overproduction of gravitons in processes such as $\gamma+\gamma \rightarrow G$, which requires $T_{r} \leq 60 \mathrm{MeV}$ for two extra dimensions [7]. In fact, the allowed $T_{r}$ ranges from $O(10) \mathrm{MeV}$ up to $O(10$ -100) $\mathrm{GeV}$ depending on the number of extra dimensions. The second important observation is that the inflaton field in these models has a natural coupling to the SM fields, which is Planck-mass-suppressed [5]. This is due to the fact that the inflaton field resides in the bulk. This helps to inflate the size of the extra dimension from its natural size $\left[(\mathrm{TeV})^{-1}\right]$ to its present millimeter size in order to maintain the hierarchy. It also solves naturally the stabilization of the size of the extra dimensions [6], and besides all, it can provide the adequate density perturbations required for the structure formation in the Universe. As a consequence, the inflaton has a decay rate into Higgs bosons, for instance given as [5]

$$
\Gamma_{\phi \rightarrow \mathrm{HH}} \sim \frac{g^{2} M^{3}}{32 \pi M_{P}^{2}},
$$

where $g$ the coupling constant; $M$ is the fundamental scale which is related to the size of the extra space $V_{n}$, and to the four-dimensional Planck mass through [4]

$$
M^{2+n} V_{n}=M_{p}^{2} .
$$

For $n \leqslant 2$ extra dimensions, $M$ can be at a TeV range. Current experimental limits from collider physics and supernova 1987A impose a bound: $M \geqslant 30 \mathrm{TeV}[4,10]$ for $n=2$.

While deriving the decay rate in Eq. (1), we have implicitly assumed that the mass of the inflaton is roughly of the order of the fundamental scale $\sim M$, in order to generate adequate density fluctuations $[5,6]$. The estimated reheat temperature of the Universe is given by $T_{r} \sim 0.1 \sqrt{\Gamma M_{p}}$ $\sim 1$ (10) $\mathrm{MeV}$, right above the temperature required for successful big-bang nucleosynthesis. Notice that this result is independent of the number of extra dimensions. It is also worth mentioning that the decay rate of the inflaton field into the relativistic particles, such as light degrees of freedom, has a similar suppression to Eq. (1). Notice two important points: in our case, the Higgs field can be treated as a heavy (nonrelativistic) particle, and the inflaton decaying into the Higgs boson is as favorable as that decaying into very light particles. This makes a difference while discussing the maximum temperature reached during the reheating era, which can be quite different from the final reheat temperature of the Universe. As the inflaton field oscillates with a decaying amplitude, the Universe is gradually filled up by the light degrees of freedom, which produces an effective temperature of the Universe which follows a different scaling relationship between the temperature and the scale factor. The temperature reaches its maximum when $a / a_{I} \sim 1.48$, where $a$ denotes the scale factor of the Universe and the subscript $I$ denotes the era when inflation comes to an end. In the large extra dimension models, the inflationary scale is determined by $H_{I} \sim M[5,6]$. After reaching the maximum temperature, it decreases as $T \sim 1.3\left[g_{*}\left(T_{m}\right) / g_{*}(T)\right]^{1 / 4} T_{m} a^{-3 / 8}$, where $T_{m}$ denotes the maximum temperature [8,9]. For $M \sim 10 \mathrm{TeV}$, the maximum temperature could reach $T_{m} \sim 10^{5} \mathrm{GeV}$ as mentioned in Ref. [9]. The basic assumption that goes behind this derivation is that the inflaton field is predominantly decaying into the relativistic species. However, this may not be the case. By reversing the argument, and, naively assuming that the inflaton decay populates only the non-relativistic degrees of freedom, one can show that the maximum temperature follows: $M \geqslant T_{m} \gg T_{r}$, but still much higher than the reheat temperature of the Universe. Note that in this case the temperature-scale factor dependence, however, follows $T$ $\propto a^{-1}$. In either case, eventually the massive particles have to decay into a radiation bath. The decay rate of these intermediate particles is now governed by their gauge couplings. If this happens, the Universe might again be populated by radiation domination while the inflaton field is oscillating. This could again raise the maximum temperature above 100 $\mathrm{GeV}$. Thus, the result apparently seems to be a robust one. This might be favorable to electroweak baryogenesis. However, it is still not clear whether the sphaleron transitions can be made useful for other sources of baryogenesis, such as leptogenesis. This is the topic we shall briefly touch upon before discussing the Affleck-Dine baryogenesis.

\section{A. Leptogenesis}

Following our previous discussion, one might suspect that the lepton number produced in the decay process of a heavy neutrino can be processed into the baryon number by anomalous $(B+L)$-violating sphaleron interactions which are in equilibrium for a temperature more than $100 \mathrm{GeV}$. However, there is a simple catch in this proposal. A singlet righthanded neutrino can naturally couple to the SM lepton doublet and the Higgs field in the following way: $h \bar{L} H N$. This leads to a potentially large Dirac mass term unless the Yukawa coupling $h \sim 10^{-12}$ or so. Moreover, now the seesaw mechanism fails to work, since the largest Majorana mass we may expect can never be larger than the fundamental scale. Therefore, given a neutrino mass $\sim h^{2}\langle H\rangle^{2} / M \sim h^{2}$ - $O$ (1) $\mathrm{GeV}$, we still have to fine-tune $h^{2} \lesssim 10^{-10}$ in order to obtain the right order of magnitude for the neutrino mass. Thus, the right-handed neutrinos, if they exist at all, are more likely to be bulk fields rather than brane fields, since in such a case the volume suppression of the bulk-brane coupling naturally provides a small coupling [11]. In any case, the decay rate of the right-handed neutrino to the SM fields is suppressed by the smallness of $h$, which gives rise to a decay 
rate that is similar to Eq. (1). This makes it extremely difficult to realize baryogenesis, because eventually when the right-handed neutrino decays into the SM fields, the background temperature is of the order of the reheat temperature $\sim O(1-10) \mathrm{MeV}$, and at this temperature the sphaleron transition is not at all in equilibrium. The sphaleron transition rate is exponentially suppressed. So, a seemingly suitable lepton number might not even get converted to the baryons to produce the desired baryon asymmetry in the Universe. Indeed, a larger reheating temperature, at least $\mathcal{O}(1$ -100) $\mathrm{GeV}$, is required to make this scenario viable [12].

On the other hand, it might be possible that sphalerons can reprocess a preexisting charge asymmetry into baryon asymmetry [13] reflected in an excess of $e_{L}$ over anti- $e_{R}$ created during inflaton oscillations. This mechanism requires that $(B+L)$-violating processes are out of equilibrium before $e_{R}$ comes into chemical equilibrium, such that the created baryon asymmetry could be preserved. Again, this has to happen during or above $100 \mathrm{GeV}$. Nevertheless, it is important to remember that the decaying inflaton field certainly injects more entropy into the thermal bath provided that the inflaton decays dominantly into the relativistic degrees of freedom. So, an initially large baryon asymmetry has to be created in order to obtain the right amount of asymmetry just before nucleosynthesis. One can easily estimate the amount of dilution that the last stages of reheating era will produce. The entropy dilution factor is given by

$$
\gamma^{-1}=\left(\frac{s\left(T_{r}\right)}{s\left(T_{c}\right)}\right)=\left(\frac{g_{*}\left(T_{r}\right)}{g_{*}\left(T_{c}\right)}\right)\left(\frac{T_{r}}{T_{c}}\right)^{3}\left(\frac{a\left(T_{r}\right)}{a\left(T_{c}\right)}\right)^{3},
$$

where $s$ is the entropy and $T_{c}$ denotes the electroweak temperature $\sim 100 \mathrm{GeV}$. For a low reheat temperature such as $T_{r} \sim 1 \mathrm{MeV}$, the above expression gives rise to $\gamma^{-1} \geq 10^{25}$. While calculating the ratio between the scale factors, we have used $T \propto a^{-3 / 8}$ and $g_{*}\left(T_{c}\right) \approx g_{*}\left(T_{r}\right)$. Therefore, including the entropy dilution factor, one may conclude that the initial $n_{b} / s$ has to be extremely large $\gtrsim 10^{15}$ in order to produce the required baryon asymmetry during nucleosynthesis, which is $n_{b} / s \sim 10^{-10}$. Such a large baryon asymmetry is an extraordinary requirement in any natural model of baryogenesis, and is almost impossible to achieve in our case.

There are a few important lessons to be learned from the above analysis. First of all, the large production of entropy during the last stages of reheating can in principle wash away any baryon asymmetry produced before the electroweak scale. The second point is that it is extremely unlikely that leptogenesis will also work because one needs to inject enough lepton asymmetry in the Universe before the sphaleron transitions are in equilibrium. The only simple choice left is to produce baryon asymmetry directly, however just before the end of reheating. The sole mechanism which seems to be doing well under these circumstances is the Affleck-Dine baryogenesis, which we shall discuss in the following section.

\section{AFFLECK-DINE BARYOGENESIS}

Affleck and Dine have proposed a beautiful scenario of baryogenesis in the context of supersymmetry [14]. A scalar condensate which carries nonzero baryonic, or/and leptonic charge survives during inflation and decays into SM fermions to provide a net baryon asymmetry. In our case, the AD field, $\chi$, is a singlet carrying some global charge which is required to be broken dynamically in order to provide a small asymmetry in the current density. This asymmetry can then be transformed into a baryonic asymmetry by a baryonviolating interaction, which we discuss later on. In order to break this $U(1)_{\chi}$ charge, we require a source term which naturally violates $C P$ for a charged $\chi$ field, and during the nontrivial helical evolution of the $\chi$ field it generates a net asymmetry in $\chi$ over $\bar{\chi}$. This necessarily has to happen after the end of inflation. Notice that in our case the initial $C P$ phase is completely arbitrary and determined during the end of the inflationary era.

We remind the readers that the inflaton energy density must govern the evolution of the Universe, and the decay products of the inflaton are also responsible for reheating the Universe. This happens once the inflaton decays before $\chi$ decays into SM quarks and leptons. This decay of $\chi$ via baryon-violating interaction generates a baryon asymmetry in the Universe which is given by

$$
\frac{n_{b}}{s} \approx \frac{n_{b}}{n_{\chi}} \frac{T_{r}}{m_{\chi}} \frac{\rho_{\chi}}{\rho_{I}} .
$$

The final entropy released by the inflaton decay is given by $s \approx \rho_{I} / T_{r}$. The ratio $n_{b} / n_{\chi}$ depends on the total phase accumulated by the $\mathrm{AD}$ field during its helical motion in the background of an oscillating inflaton field, which can be at most $\approx O(1)$. If we assume that the $\mathrm{AD}$ field is a brane-field, then the energy density stored in it can be at most $\rho_{\chi}$ $\approx m_{\chi}^{2} M^{2}$, on the other hand the energy density stored in the (bulk) inflaton field is quite large, $\rho_{I} \approx M^{2} M_{p}^{2}[5,6]$. Thus, we get the ratio $n_{b} / s \sim\left(T_{r} / M_{p}\right)\left(m_{\chi} / M_{p}\right) \approx 10^{-34}\left(m_{\chi} / M\right)$ $\ll 10^{-10}$ for $T_{r} \sim O(1-10) \mathrm{MeV}$. The conclusion of the above analysis is again disappointing, as it suggests that the $\mathrm{AD}$ baryogenesis also leads to a small $n_{b} / s$. One way to boost this ratio is to assume that the AD field resides in the bulk. In that case, one naturally enhances the ratio $\rho_{\chi} / \rho_{I}$, however keeping in mind that it is still less than 1 , in order not to spoil the successes of inflation.

Once the $\mathrm{AD}$ field is promoted to the bulk, the energy density stored in the $\mathrm{AD}$ field rises to $\rho_{\chi} \sim m_{\chi}^{2} M_{p}^{2}[5,6]$. This leads to the maximum baryon to entropy ratio,

$$
\frac{n_{b}}{s} \approx\left(\frac{T_{r}}{M}\right)\left(\frac{m_{\chi}}{M}\right) \sim 10^{-10}\left(\frac{m_{\chi}}{1 \mathrm{GeV}}\right),
$$

where we have evaluated the right-hand side for $T_{r}$ $\sim 10 \mathrm{MeV}$ and $M \sim 10 \mathrm{TeV}$. Although the mass of the AD field requires some fine-tuning up to the $C P$ phase, the above ratio can reach the observed baryon to entropy ratio quite comfortably. Notice that the actual predicted value also de- 
pends on the initial conditions on $\chi$ that may give $m_{\chi}$ more freedom. Say, for instance, if $\chi_{0} \sim M_{\mathrm{GUT}}$, we get the right $n_{b} / s$ provided $m_{\chi} \sim M$.

We have noticed earlier that due to the violation of $U(1)_{\chi}$ charge, the dynamics of the AD field generates an excess of $\chi$ over $\bar{\chi}$ fields. This asymmetry is transfered into baryon asymmetry by a baryon-violating interaction, such as $\kappa \chi Q Q Q L / M^{2} M_{P}$, however keeping $B-L$ conserved. We also assume that $\chi$ interactions to SM fields conserve $U(1)_{\chi}$ symmetry, thus the quarks and leptons must carry a nonzero global $\chi$ charge while the Higgs field does not. This avoids $\chi$ decaying into Higgs bosons which otherwise will reduce the baryonic abundance and make the above interaction the main channel for its decay. While discussing the decay rate of the $\chi$ field, one has to take into account all possible decay channels which can be of the order of thousands due to family and color freedom. On the other hand, we assume that the inflaton is decaying mainly into Higgs bosons. The final result is then given by

$$
\Gamma_{\chi} \approx\left(\frac{\kappa}{g}\right)^{2}\left(\frac{m_{\chi}}{M}\right)^{7} \Gamma_{\phi} .
$$

By taking $\kappa / g \sim O(1)$, we can insure that $\chi$ will decay along with the inflaton, provided that its mass is very close to the fundamental scale. This will certainly demand some level of fine-tuning in the parameters. We would like to mention that this is perhaps the simplest scenario one can think of for generating baryon asymmetry right before nucleosynthesis takes place. It is worth mentioning that in our model the AD field will not mediate proton decay by dimension-6 operators such as $Q Q Q L$, as long as $\chi$ does not develop any vacuum expectation value. Notice that other processes mediating proton decay, such as instanton effects, might still occur. While there is no known solution for such a potential problem, our mechanism is at least not adding any new source to proton decay. In the same spirit, one may check those operators which induce $n-\bar{n}$ oscillations. Again, effective $\Delta B=2$ operators of dimensions 9, UDDUDD, and 11, $(Q Q Q H)^{2}$, cannot be induced by integrating out $\chi$.

\section{CONCLUSION}

We have noticed that the observed baryon asymmetry in the Universe is difficult to obtain in the presence of large extra dimensions. We have pointed out that there is a seemingly simple way, if we assume that there exists a SM singlet field carrying some global $U(1)_{\chi}$ charge which lives in the bulk. The nontrivial dynamics of this field generates an asymmetry in $\chi-\bar{\chi}$ after the end of inflation, which will be transfered into a baryon asymmetry by a baryon-violating interaction. It is possible to insure that the $\mathrm{AD}$ field decays along with the inflaton such that the synthesis of the light elements can take place.

\section{ACKNOWLEDGMENTS}

The authors are grateful to R. Allahverdi, P. Binetruy, S. Davidson, and K. Enqvist for helpful suggestions. A.M. acknowledges the support of The Early Universe network HPRN-CT-2000-00152.
[1] See, for instance, E. W. Kolb and M. S. Turner, The Early Universe (Addison-Wesley, Redwood City, CA, 1990).

[2] A. D. Sakharov, Pis'ma Zh. Éksp. Teor. Fiz. 5, 32 (1967); JETP Lett. 91, 24 (1967).

[3] A. D. Dolgov, Phys. Rep. 222, 309 (1992); W. Buchmuller and S. Fredenhagen, hep-ph/0001098; A. Riotto, hep-ph/9807454; A. Riotto and M. Trodden, Annu. Rev. Nucl. Part. Sci. 49, 35 (1999).

[4] N. Arkani-Hamed, S. Dimopoulos, and G. Dvali, Phys. Lett. B 429, 263 (1998); Phys. Rev. D 59, 086004 (1999); I. Antoniadis et al., Phys. Lett. B 436, 257 (1998).

[5] R. N. Mohapatra et al., Phys. Rev. D 62, 105030 (2000).

[6] A. Mazumdar and A. Pérez-Lorenzana, Phys. Lett. B 508, 340 (2001).

[7] K. Benakli and S. Davidson, Phys. Rev. D 60, 025004 (1999); L. J. Hall and D. Smith, ibid. 60, 085008 (1999).
[8] G. F. Giudice, E. W. Kolb, and A. Riotto, Phys. Rev. D 64, 023508 (2001).

[9] S. Davidson, M. Losada, and A. Riotto, Phys. Rev. Lett. 84, 4284 (2000).

[10] See, for instance, T. G. Rizzo, Phys. Rev. D 59, 115010 (1999); G. F. Giudice et al., Nucl. Phys. B544, 3 (1999); E. A. Mirabelli et al., Phys. Rev. Lett. 82, 2236 (1999); J. L. Hewett, ibid. 82, 4765 (1999); V. Barger et al., Phys. Lett. B 461, 34 (1999); L. N. Chang et al., Phys. Rev. Lett. 85, 3765 (2000).

[11] K. R. Dienes et al., Nucl. Phys. B557, 25 (1999); N. ArkaniHamed et al., Phys. Rev. D 65, 024032 (2002); R. N. Mohapatra et al., Phys. Lett. B 466, 115 (2000).

[12] A. Pilaftsis, Phys. Rev. D 60, 105023 (2000).

[13] B. A. Campbell et al., Phys. Lett. B 297, 118 (1992).

[14] I. Affleck and M. Dine, Nucl. Phys. B249, 361 (1985). 